\title{
ENABLING FACTORS FOR IN SERVICE PROVIDER ACCESS
}

\author{
A.Jøsang*, M.G.L.Bijnagte** and G.Kleinhuis** \\ * The Norwegian University of Science and Technology, N-7149 Trondheim, Norway \\ ** KPN Research, NL-2264 XZ Leidschendam, The Netherlands
}

Key words: Intelligent networks, Service provider access, INAP, SS7, TCP/IP

Abstract: The need to offer IN services across network boundaries is expected to come as a result of the ongoing deregulation, globalisation of telecom markets and the increased mobility of users. This will lead to a pressure for a clearer separation between IN service providers and network operators allowing third party service providers open access to public networks. Some operators may want to resist this development on the grounds of avoiding competition, but we claim that maintaining this view is counterproductive in a larger perspective, and that all parties will benefit from open service provider access to public networks. This paper points out that legislation and standardisation are essential factors for making public networks open for third party IN service providers, and proposes to use the TCP/IP protocol stack for the IN service provider interface.

\section{INTRODUCTION}

Telephony services depend on service logic in several parts of the network. Even a basic call requires the prefix to be analysed in the originating exchange and the local subscriber identification to be performed in the terminating exchange. When two operators cover separate geographical areas, the mutual benefit of this type of cooperation is obvious, and interconnection agreements are relatively easy to negotiate. On the other hand, if operators compete in offering services to the same subscribers, the mutual benefit is less obvious, resulting in operators being reluctant to

The original version of this chapter was revised: The copyright line was incorrect. This has been corrected. The Erratum to this chapter is available at DOI: 10.1007/978-0-387-35581-8_35 
cooperate. This difference is for example reflected by the well defined standards for GSM roaming where mobile network operators co-operate in order to provide a seamless radio interface to their subscribers, in contrast to the almost non-existing standards for providing competing services in the same access network or geographical area.

The main characteristic of the intelligent network (IN) concept is that the logic for controlling calls and call related services is separated from the physical switching and set-up of calls. For the provision of IN services it is natural to distinguish between the network operators and the service providers although they usually are one single organisation in today's networks. The public network operator (PNO) owns and operates switches, transmission lines and subscriber access networks. Service providers own and operate data and logic for determining routing and for handling call related services, as well as supporting resources such as for example speech recognition equipment or service management tools. An organisation can play the role of PNO, of IN service provider, or both. A service provider will be called third party service provider (3PSP) when offering services through a public network owned by a different organisation.

A company that is both PNO and IN service provider would intuitively be reluctant to grant 3PSPs access to its network because it would create competition in the own network. We claim that companies maintaining this view are seeing the telecom market in a too narrow perspective. Third party service provider access to public networks will enhance the quality of telecom services in general and thereby result in increased service usage and market growth, ultimately leading to increased revenue for the network operators and service providers themselves.

In existing networks IN is usually integrated with the switching equipment of the network operator. Even when telecom companies are split into separate legal and commercial entities where the IN service provider subsidiary is different from the PNO subsidiary, it is usually only in the name. In reality, the PNO subsidiary still operates the equipment that delivers the actual IN services, whereas the service provider subsidiary is only a marketing company that in fact hires all the telecom resources needed for providing IN services from the PNO subsidiary. By opening up public networks for IN service provision, the separation has to be real, i.e. there must be a physical interface to the public network.

This paper will discuss the necessary factors for making public networks open to third party service providers. Regulation is seen as essential to overcome the competition deadlock and to provide a stable and predictable environment for new service providers. Secondly, standardisation will be essential to make open access practical. In particular we propose to let the TCP/IP protocol stack be used instead of the SS7 protocol stack in the 
interface between the PNO and the 3PSP. Finally, open IN service provider access will result in more complex networks. This will require advanced technology and an infrastructure that is sufficiently mature for mastering for example service interaction and security problems.

\section{SEEING THE BENEFITS OF OPEN SERVICE PROVIDER ACCESS}

Competition is a stimulator for innovation, increased efficiency and better services. However, this may be difficult to see for companies that try to increase their profit in the short term. In many cases it depends on the time perspective a company uses, and it is important to realise that rational behaviour in a short perspective can be counterproductive in a larger perspective and vice versa.

Today's telecommunication networks have not been designed with open service provider access in mind. Their structure reflects a monolithic enterprise approach and their ability to serve user mobility and global integration is limited. IN is partly a solution to this problem. However, if IN services cannot be provided across network boundaries, their potential will be limited. From a service quality point of view there are several aspects to consider such as for example geographical coverage, availability at any time and user friendliness, and an open interface at the service provider level will make it much easier to handle these issues in a satisfactory way.

Users should be able to choose between (IN) service providers, so that they no longer are limited to those services that their PNO can offer but are also able to use services from a 3PSP. The services offered by the 3PSP will broaden the service spectrum by offering different services or equivalent services with different quality and price. By opening up public networks for IN service provider access the incumbent PNOs will face competition in an area where they have been in a monopoly situation. However uncomfortable this initially may be, the 3PSPs will become potentially large traffic generators, so that a reduced IN service market share can be compensated by increased traffic.

As soon as one PNO has opened up for IN service provider access, all the other PNOs in the same market will be forced to do the same. A PNO that resists opening up its network will avoid competition in its own network, but will run the risk of loosing subscribers, and will at the same time loose 3PSP generated traffic to competing PNOs. The most innovative IN services will only be available through PNOs that grant 3PSPs access to their networks. It will thus be dangerous to come too late with a solution for IN service provider access. 


\section{THE REGULATORY PRESSURE}

The cooperation deadlock caused by competition can only be overcome by regulation such as for example the Directive on Interconnection [1] of the European Union which has as purpose of ensuring universal service and interoperability through the principles of Open Network Provision (ONP), and ultimately of serving the objective of economic and social cohesion and territorial equity.

The EU directive states that "fixed and mobile operators with significant market power must meet all reasonable requests for access to their network ..." (Art.4.2). The term "reasonable" means that access should be granted if it is required for offering a service, if it is technically possible, and if it does not threaten the network integrity. Guidelines for the operational aspects of network integrity are given in the recommendations [2] published by the European Committee for Telecommunications Regulatory Affairs.

Furthermore, the EU directive states that interconnection agreements between the involved parties should be non-discriminating, meaning that no discrimination in own use and third party use is allowed, and that the same conditions shall be offered to all the parties asking for interconnection (Art.6.a) Interconnection agreements shall also be transparent, meaning that the agreements must be open and made available for all interested parties (Art.6.c). Finally, interconnection agreements shall be cost-oriented, meaning that the interconnection tariffs must be based on the associated costs of offering the interconnection (Art.7.2).

Finally, the EU directive states that adherence to relevant technical standards may be made compulsory (Art.13). On this background, costorientation would imply that the additional cost for service providers when connecting to a network that deviates from existing standards has to be covered by the network operator. Non-standard solutions would in addition break the principle of non-discrimination because it for example would favour interconnecting equipment from the vendor that delivered the network equipment. In the absence of relevant standards, the principles of cost-orientation and non-discrimination are severely weakened, and regulation such as the EU directive alone will not be sufficient to give 3PSPs access to public networks. The existence of relevant standards is thus crucial, and this will be discussed below.

\section{POSSIBLE ACCESS INTERFACES}

All network elements in an IN architecture can in principle be subject to 3PSP access, but some are more suitable and natural points of 
interconnection than others. Figure 1 illustrates a typical single operator/service provider IN architecture with its principal elements.

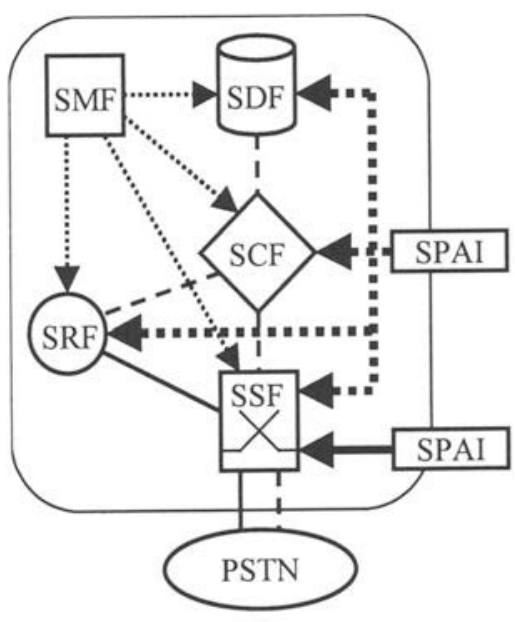

\section{Legend:}

SPAI: Service Provider Access Interface

SMF: Service Management Function

SDF: Service Data Function

SCF: Service Control Function

SRF: Specialised Resource Function

SSF: Service Switching Function

PSTN: Public Switched Telephone Network

Figure 1 Single operator IN architecture with possible SPAIs.

The required access point will in general depend on the provided service. At a high level the service types can be divided into three categories:

1. Call control services: The 3PSP provides the IN services itself. This requires control over the call by the 3PSP. In this case the 3PSP operates its own SCF and requires access either to the SSF or the SCF in the PNO domain.

2. Support services: The 3PSP can have a supporting role for the delivery of IN services, by for example providing user information in which case access to the SCF or the SDF is required, or by offering specialised resources like for example speech recognition in which case access to the $\mathrm{SCF}$ is required.

3. Management services: The 3PSP can manage services and service data on network elements in other domains, such as for example distributing or replicating data to SDFs owned by different PNOs in which case the 3PSP operates a SMF and requires access to SDFs (either directly or via the SMF in the PNO domain). The 3PSP can also have a supporting management role such as for example number portability administration, in which case the IN service provider needs an interface to the portability administrator for the communication of ported numbers.

In the next section, we give a brief overview of the available standards for interfaces offering call control services and discuss their suitability for interconnecting different domains at the IN level. 


\section{STANDARDS}

Intelligent Networks are standardised in phases, called capability sets (CS). These capability sets are indicated with CS1 [3], CS2 [4], etc. CS2 that has recently been finished (1998) -represents a superset of the functionality standardised in CS1 (1995) and is backward compatible to CS1. Within a standard various IN interfaces are standardised. Below the relevant interface standards required for call control services will be discussed.

\subsection{Interface standards for call control services}

Different options are possible for offering call control across network boundaries, as illustrated in Figure 2.

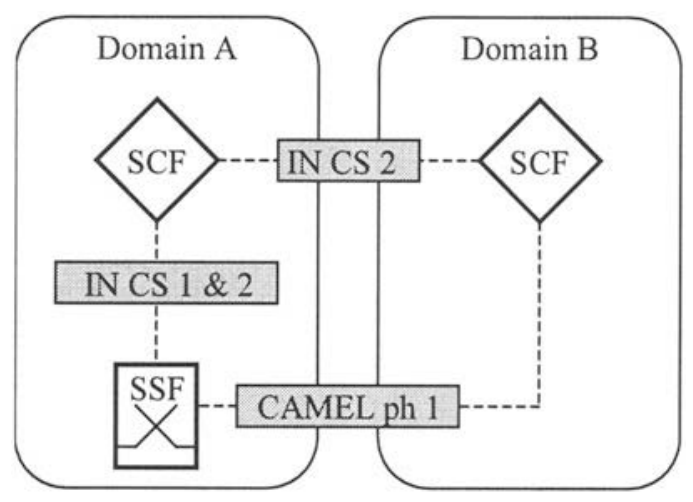

Figure 2. Interface standards for call control services.

For the interfaces required by call control services, the relevant interface standards are IN CS1 SSF-SCF interface, the IN CS2 SCF-SCF interface, and the CAMEL phase 1 interface.

\subsubsection{IN CS1\&2 for SSF-SCF interdomain connection}

The SSF-SCF interface standardised in CS1 $[3,5]$ and elaborated in CS2 $[4,6]$ allows an SCF of the IN platform to have control over the call. This interface also supports charging and user interaction functionality. Implementations of CS1 SSF-SCF are already available in public networks. Since the SSF-SCF interface is standardised for intra-networking, problems arise when using this standard over network boundaries. This standard includes a number of options that would cause obvious problems when used for inter-networking and does not address network integrity such as 
authentication and screening functions. Some INAP (Intelligent Network Application Protocol) operations can cause significant problems to the network integrity. For instance (with issuing the callGap operation) the SCF can instruct the SSFs to block the sending of IN queries which could be unacceptable. The same applies for the operation activateServiceFiltering that allows an SCF to instruct the SSFs to filter on specific servicekeys. Also the ability of (re-) setting counters and timers can cause problems. Therefore, at this moment the network integrity is not maintained if this standard is used for inter-networking.

\subsubsection{CAMEL phase 1 for SSF-SCF interdomain connection}

The CAMEL (Customised Application for Mobile network Enhanced Logic) interface [7] offers a way of interconnecting an SCF and an SSF in a mobile environment. The purpose of CAMEL is to make services from the home domain available in another domain. Phase 1 of this interface is a subset of the existing IN CS1 standard, with some mobile specific parameter extensions. Products for phase 1 have recently arrived on the market. Due to the limited functionality of phase 1 , the network integrity is less a problem since powerful operations, like call gapping, and operations for (re-)setting counters and timers are excluded. The use of the CAMEL phase 1 interface for third party service provider access has some drawbacks. In phase 1 no charging and user interaction capabilities are available. CAMEL offers limited functionality compared to the current implementations in fixed networks. This results in discriminatory conditions. Therefore, using CAMEL phase 1 for third party service provider access will probably not meet the 3PSP's (and regulatory) requirements, In addition, implementation difficulties will arise when integrating this standard with its mobile specific parameter extensions in an fixed IN network.

\subsubsection{IN CS2 for SCF-SCF interdomain connection}

The SCF-SCF interface is standardised within CS2 [4,6] for distributed service execution and can be used over network boundaries. The communication is basically between two co-operating 'service logics' in two SCFs. The SCF triggered by an SSF, called the 'controlling' SCF, initiates the communication and requests a 'supporting' SCF to perform some necessary actions, e.g. determine a routing address for a call or determine the call charge. Products with implementations of CS2 standards are not expected before the year 2000. Authentication procedures which are crucial for maintaining network integrity are specified in the CS2 SCF-SCF interface. In principle, the SCF-SCF interface can be used for 
interconnecting third party service providers, but the 3PSP will not directly control the call. The service logic in the controlling SCF has to map the information received from the supporting SCF into the operations instructing the SSF how to process the call. With the disadvantage that for almost every new service offered by a third party service provider specific service logic is needed in the SCF of the PNO. This dependence of the 3PSP on the PNO has consequences for e.g. the time to market of its services and will therefore not meet the 3PSP's requirements.

\section{ENABLING TECHNOLOGIES}

As we saw in the previous section, the current available standards are not adequate to meet the requirements of offering adequate functionality for call control and maintaining the network integrity. In order to fill this gap, we will describe additions to standards and propose technical solutions for service provider access for call control services. Primarily, additions to the current standards are required. This includes:

- The possibility to perform screening actions on the messages exchanged between the two parties. For instance per traffic relationship between an 3PSP and an PNO a screening profile should be agreed in which the allowed operations, parameters and parameter ranges are negotiated. According to this profile the screening actions will be performed.

- The mapping of network specific options within an PNO or 3PSP domain into bi-lateral agreed formats.

- The possibility to measure transaction flows between an entity in the PNO domain and an entity in the 3PSP domain. For accounting purposes information on traffic flows between an 3PSP and PNO are needed. A large number of 3PSPs may be interconnected to the PNO's IN platform, and therefore capacity has to be reserved. In order to be able to guarantee the reserved capacity, the thresholds for the volumes of traffic negotiated must be checked.

Secondly, the networks must be resistant against malicious attacks, either by external parties, or by one party trying to (whether on purpose or not) abuse the otherwise legal access to another party's network.

The problem of providing secure transactions across domain boundaries is one of the most challenging problems of distributed systems because security ultimately must be based on a common security policy that defines e.g. who is authorised and what is allowed. If different domains cannot agree on a single security policy the interaction must be subject to special agreements between domains on a pair to pair basis. In case of transnational 
service provision, problems related to international legal disputes must also be addressed.

The security aspects needed for domain interconnection include:

- Data integrity: Only authorised agents shall be able to create, delete or modify messages. Data integrity can be obtained by message authentication codes, by encryption, by authentication or by digital signatures.

- Confidentiality: Only authorised agents shall be able to read messages. Confidentiality is implemented by encryption.

- Authentication: The recipient of a message shall be able to verify the identity of the sender. Authentication is implemented by the means of protocols consisting of a sequence of messages.

- Non-repudiation: The sender of a message shall not be able to deny having sent the message. In the same way, the recipient of a message shall not be able to deny having received the message. Non-repudiation is implemented by using digital signatures.

- Accountability: It shall be possible to trace all actions to identified agents, and agents shall be made responsible for their actions.

Authentication and non-repudiation both address the same basic problem of verifying identity. In case the two interworking parties trust each other, authentication is sufficient. In case the parties do not trust each other, a third party mediator must be able to verify the identity of the sender, and that can only be done by non-repudiation based on digital signatures. Nonrepudiation is thus stronger than authentication.

In order to provide the missing functionality and security requirements, we propose the introduction of an interworking function where the functionality described above is located. Secondly we propose to use the TCP/IP protocol stack instead of SS7 as the bearer of INAP. Finally we propose to use the Secure Socket Layer protocol (SSL) [8] or its standardised version the Transport Layer Security Protocol (TLS) [9], between TCP/IP and INAP in order to obtain a secure channel between domains. These points will be described separately below.

\subsection{The interworking function}

The InterWorking Function (IWF) acts as a gateway function between the PNO's and the 3PSP's domains. Functionality missing in the standards such as screening, mapping and measuring functions are provided by this IWF. In addition the IWF should perform authentication of the entities involved (see section 6.3 below). Figure 3 illustrates how two domains interact via the interworking function. 


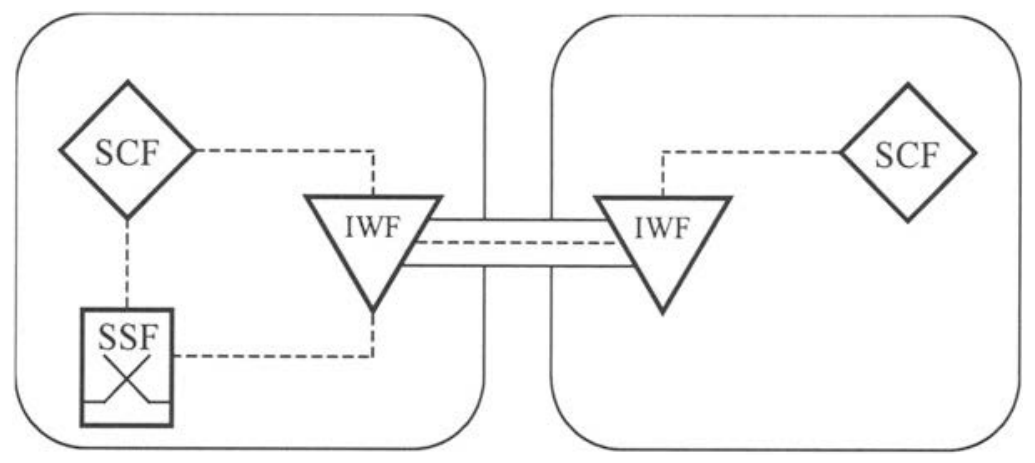

Figure 3. The positioning of the interworking function.

The interworking function is functionally located between the SSF in the 3PSP domain and the SCF in the 3PSP domain. This concept of an interworking function can be used as a gateway both in case of interconnecting between the SSF in the PNO domain and the SCF in the 3PSP domain, as in case of interconnecting between the SCF in the PNO domain and the SCF in the 3PSP domain. Since both the 3PSP and the PNO must be able to perform screening, mapping and measuring, a IWF in both domains is needed. At the physical level the implementation of this function depends on the PNO's and 3PSP's equipment, the interworking function can in principle be collocated in the SCF, as well as in the SSF. A standalone solution is also possible, when placing the IWF in a separate physical unit.

\subsection{Using TCP/IP instead of SS7}

Presently, almost all IN implementations are using INAP running over Signalling System No.7 (SS7) [10,11,12]. Using SS7 for service provider access imposes several problems. Primarily, the usage of platforms that implement the SS7 protocol stack have been limited to pure telecom applications and remain relatively expensive compared to for example TCP/IP based platforms. Secondly, the TC protocol [10] that is part of SS7 provides powerful functions that potentially could cause severe damage to a network if used inappropriately, and would make network operators more reluctant to open service provider access. Finally, using SS7 would be at odds with the trend of integrating data and telecommunications networks because SS7 is limited to the more traditional telecommunication networks.

We will see more and more applications based on TCP/IP, and the product spectrum based on this technology is much broader and have lower prices than corresponding SS7 based products. A way forward to enable third party service provider access is therefore to use INAP on top of TCP/IP 
between network operators and service providers. Figure 4 illustrates the proposed protocol architecture for open IN service provider access, and compares it with the traditional SS7 protocol architecture as well as with the OSI reference model. In addition to the TCP and IP layers, the proposed architecture includes a security layer that will be described in the next section.

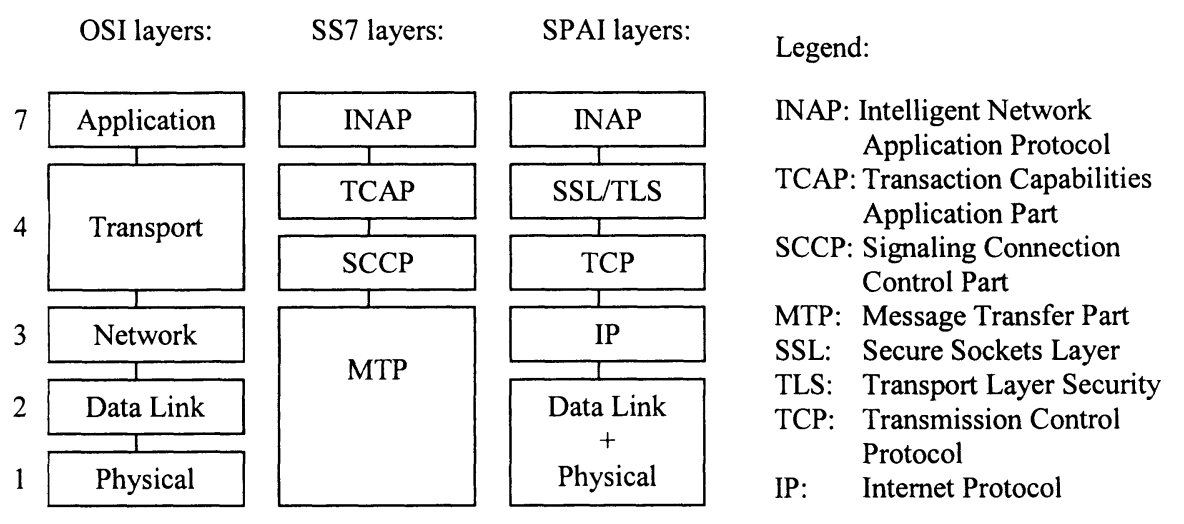

Figure 4. Proposed protocol architecture for IN service provider access.

\subsection{The secure channel}

Authentication of messages can be obtained by establishing a secure channel that is transparent to the service logic. If required, this can also give data integrity and confidentiality. The secure channel protocol must be standardised to satisfy the non-discrimination requirement. The Secure Socket Layer protocol (SSL) [8] that is already implemented in many products and its standardised version, the Transport Layer Security protocol (TLS) [9] will satisfy these requirements. TLS, which is effectively SSL 3.1, is standardised by the Internet Engineering Task force. TLS will form a protocol layer between INAP and TCP/IP in order to provide the necessary security functions.

Competition can be an incitement to act selfishly, and is therefore likely to create distrust. Regulation alone is not enough to verify that the parties fulfil their obligations, and security mechanisms can be used for this purpose. Security mechanisms can be described as a method to propagate trust from where it exists to where it is needed. Something therefore has to be trusted in the first place.

Two companies that trust each other can exchange keys and establish a secure interconnection without referring to any third party. If on the other hand the parties do not trust each other, the domains must at least agree on a 
common trusted third party (TTP) that is trusted to issue certified cryptographic keys. Using these keys, the parties can establish secure channels between each other in order to provide IN services across domain boundaries. This is illustrated in Figure 5 where the trusted third party function (TTPF) is used to distribute certified keys. Based on certificates from the TTPF, the two domains are able to set up a secure channel and interwork to provide IN services.

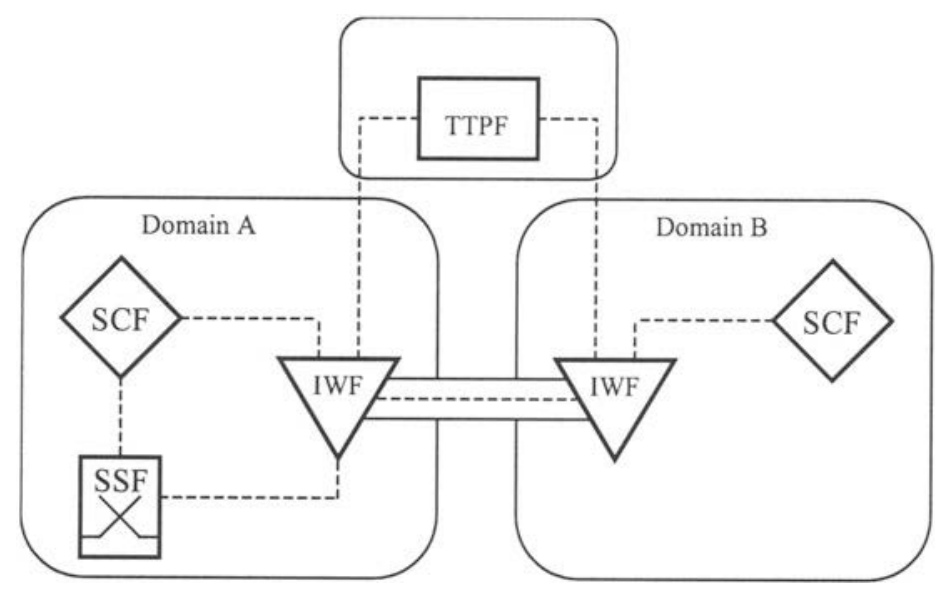

Figure 5. Interworking with a Trusted Third Party Function.

In the framework of public key cryptography, a certificate is a digital signature on an entity's public key, issued by a TTP, guaranteeing the binding between the public key and the identity of its owner. In that way, a 3PSP can use its certified public key to sign commands sent to the PNO and thereby provide the PNO the ability to perform authentication and to assure the PNO that the commands can be traced and not subsequently be repudiated. The X.509 standard [13] for exchanging public key certificates is already used by commercial TTPs, and is the natural choice for establishing a framework for open IN service provider access.

A domain interworking scheme including a trusted third party has already been established in the USA in connection with the regional local number portability. In order to have a fair and equitable solution, the industry has adopted regional neutral administrators, called NPAC (Number Portability Administration Center) that must not be in business as a network operator. There are 7 NPACs in the USA, one per regional Bell operating company area. The role of the NPAC is to receive numbering porting requests from various local network operators through a standard formatted interface called the NPAC Interface Requirement Document [14]. The NPAC coordinates the number exchange in an automated and timely fashion between the 
network operators, and has the responsibility to maintain a master database for the assigned region.

\section{MASTERING THE INCREASED COMPLEXITY}

By allowing telecom users to buy services from different operators and service providers the overall complexity increases. By considering a normal two-party call in a traditional single operator environment there is always a single point of control in each side of the call so that for example feature interaction problems can be controlled to a certain degree. In a multi service provider environment the level of feature interaction control that any operator or service provider can offer is drastically reduced unless a solution for service integration can be found.

Reliable billing is essential for any player in the telecom operator and service market. In an environment with multiple service providers, operators and service providers must be able to log and agree on what network resources have been used by whom. Billing records will have to be collected from many different sources before being presented to the end user, and internal billing between operators and service providers must be done accordingly.

In a single operator environment users had a single point of contact for requests and complaints. Unless operators and service providers can agree on a co-ordinated customer interface for reporting technical problems, the users risk being the victims of a responsibility vacuum.

Handling the problems mentioned above could in fact become separate commercial activities called for example service integrator or service mediator.

\section{CONCLUSION}

Considering the way in which telecommunication networks have developed in the past 100 years where services and networks have always been integrated third party service provider access does not come naturally. Although technically challenging, it is highly desirable in order to increase competition and remove old monopolistic barriers. This paper has discussed some of the enabling factors for this development. Services can be divided into different categories such as management, support services and call control services, where this paper has focused on the latter. The major problem when interconnecting different domains is to maintain network integrity. For this purpose we have proposed a technical solution which uses 
the TCP/IP protocol architecture instead of SS7 for INAP, and which includes a secure protocol layer between the INAP and TCP/IP protocol layers that can be used to establish secure channels between domains. In addition we have proposed an interworking function that acts as a gateway between domains. Apart from these technical solutions provisions need to be made by the public network operators and the third party service providers in order to manage the increased complexity e.g. billing and feature interaction.

\section{REFERENCES}

1. Directive 97/33/EC of the European Parliament and of the Council on interconnection in telecommunications with regard to ensuring universal service and interoperability through application of the principle of open network provision (ONP). The European Commission, 1997.

2. Recommendation Rec(98)01 - Network integrity, European Conference Of Postal and Telecommunications Administrations/European Committee for Telecommunications Regulatory Affairs (CEPT/ECTRA), 1998.

3. Recommendation series Q.1210 - Intelligent network capability set 1 (CS-1), International Telecommunication Union, Telecommunication Standardization Sector (ITU-T), 1995.

4. Recommendation series Q.1220 - Intelligent network capability set 2 (CS-2), International Telecommunication Union, Telecommunication Standardization Sector (ITU-T), 1998.

5. ETS 300374 - Intelligent Network (IN); Intelligent Network Capability Set 1 (CS1); Core Intelligent Network Application Protocol (INAP); Part 1: Protocol specification. European Telecommunications Standards Institute (ETSI), 1994.

6. ETS 301140 - Intelligent Network (IN); Intelligent Network Capability Set 2 (CS2); Part 1: Protocol specification. European Telecommunications Standards Institute (ETSI), 1999.

7. ETS301 152-1- Intelligent Network (IN); Intelligent Network Capability Set 1 Extension; Intelligent Network Application Protocol (INAP); Part 1: Protocol specification for CAMEL Phase 1. European Telecommunications Standards Institute (ETSI), 1997.

8. The SSL 3.0 Protocol, Netscape Communications Corp. Feb.9, 1995.

9. The TLS Protocol, RFC2246, Internet Engineering Task Force, January 1999.

10. Recommendation Q.773- Specification of Signalling System No.7; Transaction Capabilities formats and coding, International Telecommunications Union, Telecommunication Standardization Sector (ITU-T), 1993.

11. Recommendation Q.711 to Q.714 and Q.716 - Specification of Signalling System No.7; Signalling Connection Control Part (SCCP), International Telecommunications Union, Telecommunication Standardization Sector (ITU-T), 1993.

12. Recommendation Q.701 to Q.708 - Specification of Signalling System No.7; Message Transfer Part (MTP), International Telecommunications Union, Telecommunication Standardization Sector (ITU-T), 1993.

13. Recommendation X.509 - The Directory Authentication Framework, International Telecommunications Union, Telecommunication Standardization Sector (ITU-T), 1993.

14. NPAC SMS Interoperable Interface Specification, NANC version 1.7. Illinois Commerce Commission, 1997. 